\title{
Responding on a multiple variable-time variable-time schedule as a function of component duration
}

\author{
FRANCES K. McSWEENEY \\ Washington State University, Pullman, Washington 99164
}

\begin{abstract}
Five pigeons pecked lighted keys for food reinforcers delivered by a multiple variable-time 30 -sec variable-time 2-min schedule. The duration of the components varied from $5 \mathrm{sec}$ to $16 \mathrm{~min}$. The rate of responding generated by the more favorable component schedule decreased as component duration increased to an intermediate value and then increased with additional increases in duration. The decrease confirmed a prediction of additive theories of behavioral contrast. The rate of responding generated by the less favorable component did not increase as component duration increased. This decrease may represent a floor effect or it may violate a prediction of one additive theory of contrast.
\end{abstract}

Gamzu and Schwartz (1973) placed hungry pigeons on several multiple variable-time variable-time (mult VT VT) schedules of food presentations. That is, they present food freely after variable intervals of time regardless of what the subjects did. Different rates of food presentation occurred in the presence of different color lights which alternated on a standard response key. The pigeons pecked the key at a high rate when it was illuminated by the stimulus that signaled the higher rate of food presentation (the more favorable stimulus). They pecked at a lower rate during the stimulus that signaled the lower rate of food (the less favorable stimulus). And they pecked both stimuli at a lower rate when the stimuli signaled equal rates of food presentation.

Several recent, additive, theories have related these responses to the behavioral contrast which occurs when pigeons respond on multiple variable-interval variable-interval (mult VI VI) schedules of reinforcement (e.g., Gamzu \& Schwartz, 1973; Hearst \& Jenkins, 1974; Rachlin, 1973). Mult VI VI schedules are similar to mult VT VT schedules, except that food is not given freely. The subjects must perform a particular response to obtain it. Positive behavioral contrast refers to an increase in the rate of responding in one component which occurs when the rate of reinforcement obtained from the other component decreases. Negative behavioral contrast refers to a decrease in the rate of responding which occurs when the other rate of reinforcement increases.

The additive theories differ in detail, but they all

The author wishes to thank K. Kearney, G. Grammer, and $P$. Campbell for their assistance in running the subjects. Reprints may be obtained from the author, Department of Psychology, Washington State University, Pullman, Washington 99164. agree that positive behavioral contrast occurs when biological responses add to instrumental responses. Instrumental responses are generated by the relation between the subject's responses and the food they obtain. Biological responses are generated by some aspect of the relation between the discriminative stimuli and the food that is given in their presence. According to these theories, mult VT VT schedules generate biological, but not instrumental, responses. They arrange a relation between stimuli and food: particular rates of food presentation occur in the presence of particular stimuli. But they do not arrange a relation between the subject's responses and food: food is given freely. Mult VI VI schedules generate both biological and instrumental responses. Particular rates of food presentation occur in the presence of particular stimuli, and particular responses produce food. Positive behavioral contrast occurs when the rate of biological responding generated by a mult VI VI schedule increases, but the rate of instrumental responding remains constant.

For example, Rachlin's theory argues that biological responses are excited by transitions from stimuli which signal lower rates of reinforcement to stimuli which signal higher rates (Rachlin, 1973). The number of biological responses that are excited by a stimulus transition is maximal immediately after the transition. The effect disappears rapidly, possibly within $8 \mathrm{sec}$, certainly within $16 \mathrm{~min}$ (Green $\&$ Rachlin, 1975). No biological responses are generated by mult VI VI schedules which supply equal rates of reinforcement in the two components. No transitions between stimuli of different value occur. Biological responses will be excited if the rate of reinforcement obtained from one component remains constant while the rate obtained from the other component decreases. The appearance of the 
stimulus which signals the constant component now represents a transition from a less favorable to a more favorable stimulus. Biological responses will add to the rate of instrumental responding emitted during this component if the experimenter records them. The increase in response rate will be labeled positive behavioral contrast.

The additive theories differ in their explanations of negative contrast. Rachlin's theory, which will be examined here, states that negative contrast occurs when biological responses subtract from instrumental responses. He argues that the transition from a stimulus which signals a higher rate of reinforcement to one which signals a lower rate inhibits biological responding. Inhibited biological responses may subtract from instrumental responses to produce the decrease in response rate that is labeled negative contrast. Again, the effect of a stimulus transition is transient.

Two predictions emerge from these theories. First, all of the additive theories predict that the factors which control the size of positive behavioral contrast should be the same as the factors which control the rate of responding emitted during the more favorable component of a mult VT VT schedule. The responses that occur on mult VT VT schedules and the responses that produce positive behavioral contrast are both examples of the same biological responses. Several past studies may have shown that component duration influences the size of positive contrast, (e.g., Bloomfield, 1967; Catania \& Gill, 1964; Nevin \& Shettleworth, 1966). If these studies are correct, then the additive theories should predict that component duration will control the rates of responding emitted on the more favorable components of mult VT VT schedules.

Second, Rachlin's theory directly predicts that component duration should control the rates of responding emitted on both components of a mult VT VT schedule. The theory predicts that the rate of responding emitted during the more favorable component of a mult VT VT schedule should decrease with increases in component duration, and that the rate of responding emitted during the less favorable component should increase with increases in component duration. The more favorable component should excite biological responding because its presentation represents a transition from a stimulus which signals a lower rate of reinforcement to one which signals a higher rate. The less favorable component should inhibit biological responding because its presentation represents a transition from a stimulus which signals a higher rate of reinforcement to one which signals a lower rate. Shorter components should sample the period of maximal excitation or inhibition of biological responding for a larger proportion of their total duration: the excita- tion or inhibition of biological responding is maximal immediately after a stimulus transition. Therefore, excited biological responses should produce a higher rate of responding during the more favorable components when component durations are short than when they are long. Inhibited biological responses should produce a lower rate of responding when component durations are short, if any other responses are emitted.

The present study examines the changes in the rates of responding generated by the components of a mult VT VT schedule as a function of component duration. Spealman (1976) showed that the rates of responding generated by the more favorable components of mult VT VT schedules do vary inversely with component duration. But this study is incomplete. First, Spealman varied component duration only up to $5 \mathrm{~min}$. A related study by Green and Rachlin (1975) suggests that the effect of a stimulus transition may last 8 or $16 \mathrm{~min}$. Second, Spealman did not test Rachlin's prediction for the less favorable component schedule. Almost no responding occurred during this stimulus in his study. Therefore, no increase in response rate with increases in component duration could be detected.

\section{METHOD}

\section{Subjects}

Five naive homing pigeons, maintained at $80 \%$ to $85 \%$ of their free-feeding body weights, served as subjects.

\section{Apparatus}

The apparatus was a standard three-key Grason-Stadler experimental enclosure for pigeons, Model E6446C, enclosed in a Grason-Stadler sound-attenuating chamber, Model E3125A-300. Electromechanical equipment located in another room presented the experimental events.

\section{Procedure}

All of the subjects were trained to eat from the food magazine. Then the subjects were placed on a mult VT $30-\mathrm{sec}$ VT 2 -min schedule. That is, food was presented freely every $30 \mathrm{sec}$, on the average, during a red light, and every $2 \mathrm{~min}$, on the average, during a white light. For four of the subjects, the lights alternated every $5 \mathrm{sec}$ on the central response key, located directly above the food magazine. Each subject continued to respond on this schedule until responding had stabilized. Then the component duration was changed to $30 \mathrm{sec}$. The $30-\mathrm{sec}$ component duration was followed by $60-\mathrm{sec}, 4-\mathrm{min}$, and 16-min component durations. The sequence of increasing component durations was followed by the same sequence conducted in order of decreasing duration. The stimulus which had signaled the higher rate of food presentations now signaled the lower rate, and vice versa. The fifth subject, 2461 , responded on the schedules in order of decreasing, then increasing, component duration.

Each component duration was presented until responding had stabilized for each subject. Responding was considered to be stable when the rates of responding emitted during the last five sessions for which each schedule was presented fell within the range of the rates emitted during the earlier sessions. The number of sessions conducted for each subject and component duration appear in Table 1. 
Table 1

Number of Sessions Conducted for Each Subject on Each Schedule

\begin{tabular}{cccccc}
\hline $\begin{array}{c}\text { Component } \\
\text { Durations }\end{array}$ & 3 & 2457 & 2452 & 2458 & 2461 \\
\hline \cline { 2 - 6 } $5 \mathrm{sec}$ & 37 & 36 & 36 & 35 & 36 \\
$30 \mathrm{sec}$ & 21 & 21 & 25 & 21 & 22 \\
$60 \mathrm{sec}$ & 21 & 19 & 14 & 18 & 18 \\
$4 \mathrm{~min}$ & 20 & 23 & 23 & 23 & 22 \\
$16 \mathrm{~min}$ & 15 & 15 & 16 & 16 & 16 \\
$16 \mathrm{~min}$ & 41 & 41 & 48 & 41 & 22 \\
$4 \mathrm{~min}$ & 28 & 28 & 21 & 28 & 27 \\
$60 \mathrm{sec}$ & 18 & 18 & 18 & 18 & 20 \\
$30 \mathrm{sec}$ & 19 & 19 & 20 & 20 & 28 \\
$5 \mathrm{sec}$ & 20 & 18 & 18 & 21 & 22 \\
\hline
\end{tabular}

Sessions were conducted daily, 6 or 7 times per week. The color of the key light which began the session was determined randomly. Each session terminated when 405 -sec food prsentations had occurred. Food presentations were scheduled by a 12-interval Fleshler and Hoffman series (Fleshler \& Hoffman, 1962). The key lights were extinguished and pecks were not recorded during food presentation, but the component timer did not stop. All food presentations were $5 \mathrm{sec}$ long regardless of whether a component transition occurred during them.

\section{RESULTS}

Figure 1 presents the medians of the rates of responding generated by the two component schedules plotted as a function of the component duration in seconds. The component durations, which appear on the abscissa and which were used to calculate response rates, are the time for which the component was actually available to the subject. That is, they are the programmed component durations minus the time for which the magazine was presented. The medians are the medians of the rates generated over the last five sessions for which each component duration was presented. The points obtained from the two presentations of each component duration have been averaged because hysteresis did not occur. The first five set of axes represent the data from individual subjects. The sixth set of axes represents the medians of the rates generated by all subjects. The filled circles represent the responding generated by the VT 30-sec component. The open circles represent the responding generated by the VT 2 -min component.

The subjects did respond on the components of the mult VT VT schedules; and they responded at a higher rate during the stimulus which signaled the higher rate of food presentation. Four of the five subjects also responded at higher rates when the components were 5 to $30 \mathrm{sec}$ long, than when they were 60 or $240 \mathrm{sec}$ long. However, the rates of responding generated by the VT $30-\mathrm{sec}$ schedule increased again as the component duration increased from 240 to $960 \mathrm{sec}$ for all four of the subjects that did respond. And the rate of responding generated by the VT 2 -min schedule varied directly with variations in the rate of responding generated by the other component schedule. The Spearman rank correlation coefficients for the two rates responding were .60 , $.90,1.00, .90, .73$, and .68 for the five subjects, and for the median for all subjects. The rates of responding generated by both component schedules were lower than the rates reported by Gamzu and Schwartz (1973).

\section{DISCUSSION}

The data confirm one prediction of the additive theories. At least one factor which controls the size of positive behavioral contrast, component duration, also controls the rate of responding emitted during the more favorable component of a mult VT VT schedule. The data also partially confirm a prediction of Rachlin's theory. The rate of responding emitted during the more favorable, VT $30-\mathrm{sec}$, component did decrease as component duration increased at least up to an intermediate value. However, the data fail to support Rachlin's theory in two ways. First, the rate of responding emitted during the VT $30-\mathrm{sec}$

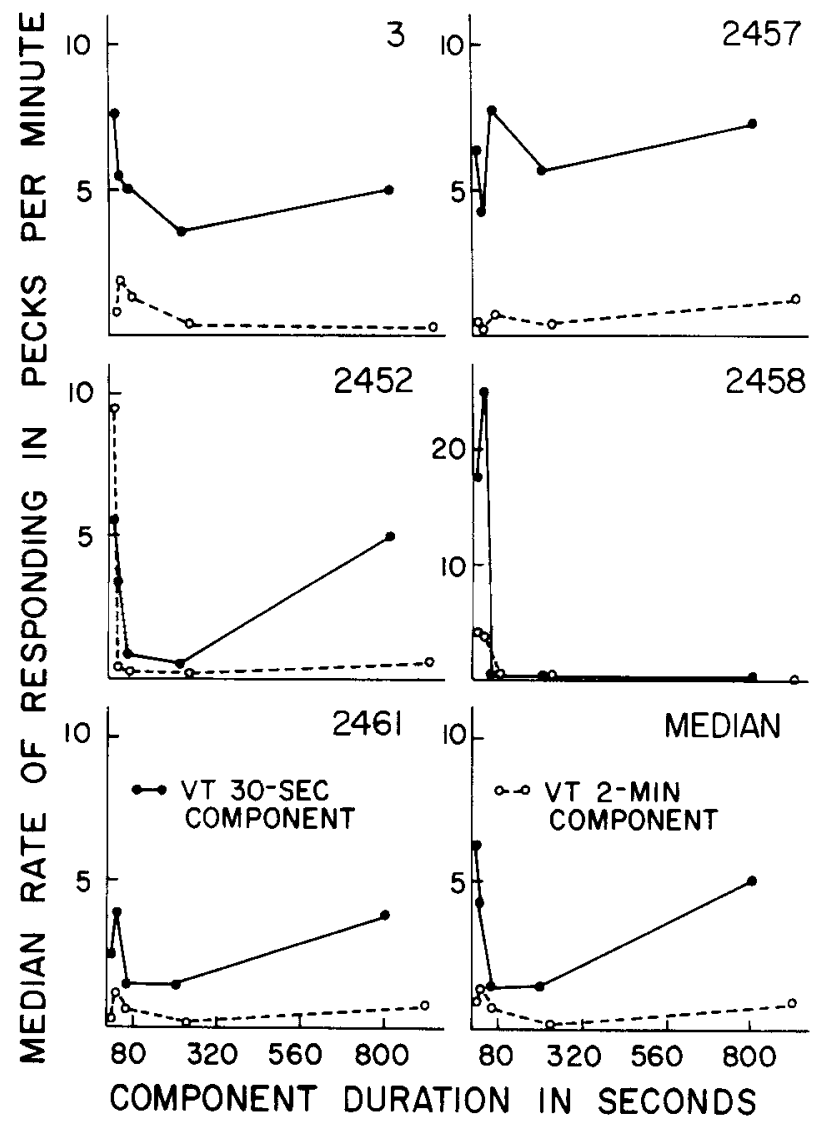

Figure 1. Median rates of responding emitted during the components of the multiple variable-time variable-time schedule as a function of component duration, for each subject and for the median of all subjects. 
component increased with further increases in component duration. Second, the rate of responding emitted during the less favorable, VT 2-min component, did not increase with increases in component duration.

The increase in the rates of responding emitted during the VT 30-sec component should be replicated, and changes in the size of positive behavioral contrast with changes in component duration should be reexamined. None of the experiments which studied contrast varied component duration to $16 \mathrm{~min}$. Therefore, it is not known whether a similar increase in the size of behavioral contrast would be found.

The increases should be investigated because they have important implications for the additive theories. All of the additive theories would be incorrect or incomplete if the increases occurred when subjects responded on mult VT VT schedules but not when they responded on mult VI VI schedules. The additive theories predict that the rates of responding generated by the two schedules should vary in the same way. Rachlin's theory would be incomplete even if the increase occurred for both schedules. The theory predicts a continuous decrease in the rate of responding emitted during the more favorable component of both schedules with increases in component duration.

The failure to find a systematic increase in the rate of responding generated by the less favorable component of the mult VT VT schedule with increases in component duration deserves further study. It may represent a floor effect. That is, the rate of responding generated by the VT 2-min schedule may have been too low for an orderly change in the rate to be detectable, or it may disconfirm Rachlin's theory of negative contrast. As argued earlier, Rachlin's theory predicts that the rate of responding generated by the less favorable component of a mult VT VT schedule should increase with increases in component duration, if any responding occurs. The theory makes this prediction because it states that the stimulus which signals the less favorable component schedule is inhibitory and that inhibition decreases with increases in time since the beginning of the component. The failure to find an increase in response rate might occur because the less favorable stimulus is not inhibitory or because the inhibitory effect does not decrease over time. Experiments should determine whether the stimulus which signals the less favorable component of a mult VT VT schedule is inhibitory (e.g., Rescorla, 1969).

\section{REFERENCES}

Bloomfield, T. M. Behavioral contrast and relative reinforcement frequency in two multiple schedules. Journal of the Experimental Analysis of Behavior, 1967, 10. 151-158.

Catania, A. C., \& Gill, C. A. Inhibition and behavioral contrast. Psychonomic Science, 1964, 1, 257-258.

Fieshler, M., \& Hoffman, H. S. A progression for generating variable-interval schedules. Journal of the Experimental Analysis of Behavior, 1962, 5, 529-530.

GAMzU, E., \& SchWARTz, B. The maintenance of key pecking by stimulus-contingent and response-independent food presentation. Jou mal of the Experimental Analysis of Behavior. 1973, 19. 65-72.

Green, L.. \& Rachlin. H. Economic and biological influences on a pigeon's key peck. Joumal of the Experimental Analysis of Behavior. 1975, 23. 55-62.

HeARST, E., \& JENkINS, H. M. Sign-tracking: The stimulus-reinforcer relation and directed action. Austin. Tex: Psychonomic Society, 1974.

Nevin, J. A., \& Shettleworth, S. J. An analysis of contrast effects in multiple schedules. Journal of the Experimental Analysis of Behavior. 1966. 9. 305-315.

RAchlin, H. C. Contrast and matching. Psychological Review'. 1973. 80. 217-234.

Rescorla, R. A. Pavlovian conditioned inhibition. Psychological Bulletin, 1969, 72, 77.94.

Spealman, R. D. Interactions in multiple schedules: The role of the stimulus-reinforcer contingency. Journal of the Experimental Analysis of Behavior, 1976, 26. 79-93.

(Received for publication August 17, 1976; revision accepted February 13, 1977.) 